\title{
Atmospheric tomography with separate minimum variance laser and natural guide star mode control
}

\author{
L. Gilles ${ }^{1}$ and B. L. Ellerbroek ${ }^{1}$ \\ ${ }^{1}$ Thirty Meter Telescope Observatory Corporation, 1200 E. California Blvd., Mail-Code 102-8, \\ Pasadena, California 91125, USA
}

\begin{abstract}
This paper introduces a novel, computationally efficient, and practical atmospheric tomography wavefront control architecture with separate minimum variance laser and natural guide star mode estimation. The architecture is applicable to all laser tomography systems, including multi conjugate adaptive optics (MCAO), laser tomography adaptive optics (LTAO), and multi object adaptive optics (MOAO) systems. Monte Carlo simulation results for the Thirty Meter Telescope (TMT) MCAO system demonstrate its benefit over a previously introduced "ad hoc" split MCAO architecture, calling for further in-depth analysis and simulations over a representative ensemble of natural guide star (NGS) asterisms with optimized loop frame rates and modal gains.
\end{abstract}

\section{Introduction}

An integral part of next generation ground-based astronomical telescopes are laser guide star (LGS) adaptive optics (AO) systems. Many systems will utilize multiple sodium LGSs in order to implement multi conjugate (MCAO), laser tomography (LTAO) and multi object adaptive optics (MOAO) [1]. The challenging science programs envisioned for these telescopes pose tight requirements on the $\mathrm{AO}$ system in terms of optical throughput, wavefront quality, image jitter, enclosed energy, and sky coverage. Robust and computationally efficient Strehl optimal tomographic wavefront control techniques will therefore be preferred on these systems compared to conventional least-squares methods.

Based on the separation principle of control theory, atmospheric tomography has been described by the astronomical AO community as a two-step process consisting of tomographic wavefront estimation, followed by deterministic control via least-squares fitting of this estimate onto deformable mirrors (DMs). Two different approaches have so far been pursued to solve the wavefront estimation step: (i) optimal solution given by Kalman filtering (which incorporates atmospheric dynamics) [2], and (ii) sub-optimal solution (without atmospheric dynamics) given by minimum variance filtering embedded in a pseudo open loop (POL) controller [3]. While the former approach is optimal, no computationally efficient algorithms have been developed so far for its realtime implementation on high-order, high bandwidth $\mathrm{AO}$ systems like those planed for future extremely large telescopes. This paper is concerned with the latter approach, which benefits from much progress toward the development of a practical MCAO real-time control architecture for the Thirty Meter Telescope (TMT) using computationally efficient algorithms [3]. For laser tomography systems, the estimation step is performed from a mixture of laser and natural guide star (LGS and NGS) wavefront sensor (WFS) measurements, the latter being required to estimate a few number of low-order atmospheric modes invisible to the tip/tilt (TT)-removed LGS WFSs on account of the LGS position uncertainty [4]. The LGS and NGS measurements have very different spatio-temporal characteristics. The former are high-order, high-bandwidth, TT-removed, and are associated with a fixed guide star geometry. The latter are low-order, low-bandwidth, and are associated with a variable guide star geometry.

In the standard formulation of minimum variance wavefront estimation, the many LGS and few NGS measurements are concatenated into a single vector and a joint (integrated) estimation is performed. While this approach is applicable to all laser tomography systems, practical considerations indicate that a split architecture with separate LGS and NGS control loops is

This is an Open Access article distributed under the terms of the Creative Commons Attribution-Noncommercial License, which permits unrestricted use, distribution, and reproduction in any noncommercial medium, provided the original work is properly cited. 
preferred. A step toward the development of such an architecture was recently made by the authors by introducing a scheme applicable to MCAO, featuring minimum variance estimation from LGS WFS measurements and "ad hoc" least-squares modal estimation from NGS WFS measurements of TT and 3 quadratic field distortion modes on 2 DMs at different conjugate ranges [3]. Control of the NGS modes is now a low dimensional problem, so that the modal NGS estimator and servo bandwidths can be rapidly updated when the NGS asterism changes. While the performance of such a scheme has been evaluated under some (possibly mild) approximations in recent sky coverage simulations [5], further improvement is still possible with optimal NGS modes tailored to each specific NGS asterism and turbulence conditions, and an optimal NGS estimator. This paper demonstrates that such elements exist and can be implemented efficiently. The strengths of this approach are: (i) optimal NGS modes estimated by a minimum variance estimator, (ii) analytical equivalence to the standard formulation of minimum variance wavefront estimation, and (iii) full applicability to all MCAO/LTAO/MOAO systems. Monte Carlo simulation results for the TMT Narrow Field Infrared Adaptive Optics System (NFIRAOS) demonstrate that this novel architecture may provide significant performance improvement over the previously introduced "ad hoc" split MCAO architecture.

The paper is organized as follows. Section 2 provides a brief review of the standard formulation of minimum variance tomographic wavefront estimation. The previously introduced "ad hoc" split MCAO architecture is then briefly reviewed in Section 3. Section 4 is devoted to the derivation of the novel architecture with optimal NGS modes and minimum variance estimators on both LGS and NGS loops. Monte Carlo simulation results for NFIRAOS illustrating the comparative performance of the 3 control architectures with a common tomography solver consisting of 30 iterations of the Conjugate Gradient (CG) algorithm are given in Section 5. Finally, conclusions are drawn in Section 6.

\section{Standard Formulation of Minimum Variance Wavefront Estimation}

Minimum variance tomographic wavefront estimation is based upon the following linearized open loop (OL) or POL WFS measurement model:

$$
s^{O L}(n)=G_{x} x(n)+\eta(n), \quad s^{P O L}(n)=s^{C L}(n)+G_{a} a(n),
$$

where $s^{O L / C L}$ denotes the concatenated vector of open loop (OL)/closed loop (CL) LGS and NGS WFS measurements, $G_{x}$ denotes the block-structured turbulence-to-WFS influence matrix mapping volume turbulence to measurements, $G_{a}$ denotes the DM-to-WFS influence matrix mapping DM commands to measurements, $x$ is the concatenated vector of atmospheric turbulence with blockdiagonal covariance matrix $C_{x}$, and $\eta$ the concatenated vector of measurement noise with blockdiagonal sparse covariance matrix $C$. All WFS-related quantities can be partitioned into LGS and NGS components as follows:

$$
s^{(P) O L}=\left(\begin{array}{c}
s_{\lg s}^{(P) O L} \\
s_{n g s}^{(P) O L}
\end{array}\right), \quad G_{x}=\left(\begin{array}{l}
G_{x, \lg s} \\
G_{x, n g s}
\end{array}\right), \quad G_{a}=\left(\begin{array}{l}
G_{a, \lg s} \\
G_{a, n g s}
\end{array}\right), \quad C=\left(\begin{array}{cc}
C_{\lg s} & 0 \\
0 & C_{n g s}
\end{array}\right) .
$$

Note that $G_{x, \lg s}$ includes a gradient TT and differential focus (DF) removal projector in its range space to account for the effect of LGS position uncertainty and spatial fluctuations in the sodium layer on the scale of the LGS asterism [6], and has thus the form sparse plus low-rank (the rank of the low-rank term being equal to $3 N_{\lg s}-1$, where $N_{\lg s}$ denotes the total number of LGSs). Note also that $C_{\lg s}$ is generally not a constant multiple of the identity due to LGS perspective elongation 
arising with continuous wave $(\mathrm{CW})$ guide star lasers. The minimum variance solution of the forward model (1) is expressed in Wiener form as follows:

$$
\hat{x}=C_{\mathrm{o}} b^{(P) O L}
$$

where $b^{(P) O L}=G_{x}^{T} C^{-1} s^{(P) O L}$ and $C_{\mathrm{o}}=A^{-1}$ with $A=G_{x}^{T} C^{-1} G_{x}+C_{x}^{-1}$ denotes the covariance matrix of the estimation error $\varepsilon=x-\hat{x}$. In terms of their LGS and NGS components, the tomography matrix $A$ and vector $b^{(P) O L}$ take the following form:

$$
\begin{array}{cll}
A=A_{l g s}+A_{n g s}, & b^{(P) O L}=b_{\lg s}^{(P) O L}+b_{n g s}^{(P) O L}, & A_{\lg s}=G_{\lg s}^{T} C_{\lg s}^{-1} G_{\lg s}+C_{x}^{-1}, \\
A_{n g s}=G_{n g s}^{T} C_{n g s}^{-1} G_{n g s,} & b_{\lg s}^{(P) O L}=G_{\lg s}^{T} C_{\lg s}^{-1} s_{\lg s}^{(P) O L}, & b_{n g s}^{(P) O L}=G_{n g s}^{T} C_{n g s}^{-1} s_{n g s}^{(P) O L} .
\end{array}
$$

For high-order systems, $C_{\mathrm{o}}$ is prohibitively expensive to compute explicitly, store and apply in realtime. Instead, the computationally efficient Conjugate Gradient (CG) algorithm can be applied to solve the tomography matrix system $A x=b^{(P) O L}$. This approach is practical provided (i) the previous-frame solution is used as initial guess to the current frame CG iterations, and (ii) application of $C_{x}^{-1}$ on a vector is computed either in the Fourier Domain (which allows to use the correct atmospheric power spectral density (PSD) and outer scale), or as a sparse curvature squared (i.e. biharmonic) operator in the Spatial Domain, an approximation which has been shown to have negligible impact upon performance [7]. We will adopt the latter option since it avoids the Fast Fourier Transforms (FFTs) and can be implemented on smaller and non-square tomography grids. Note that in practice, the NGS measurements $s_{n g s}^{(P) O L}$ are noisy and low frame rate, and must therefore be low-pass filtered prior to their concatenation to the high frame rate LGS measurements.

In the z-domain, the overall wavefront control architecture including temporal filtering is essentially described by the following equations:

$$
y(z)=H_{x} \hat{x}(z), \quad a_{o}(z)=F y(z), \quad e(z)=a_{o}(z)-a(z), \quad a(z)=g(z) e(z),
$$

where $F=\left(H_{a}^{T} \mathrm{~W} H_{a}\right)^{-1} H_{a}^{T} \mathrm{~W}$ denotes the DM fitting operator (block-diagonal for MOAO), $H_{x}$ and $H_{a}$ are the ray-tracing matrices along the fitting directions for point sources at infinite range, $\mathrm{W}$ is the block-diagonal fitting weighting matrix, and $g(z)$ are the temporal filters. Here again, for high-order systems, the inverse DM cross-coupling matrix $\left(H_{a}^{T} \mathrm{~W} H_{a}\right)^{-1}$ is prohibitively expensive to store and apply in real-time (at least for MCAO on account of its non block-diagonal structure). Instead, the CG algorithm can be used to provide an approximate solution to the fitting matrix system.

A block diagram illustrating the overall architecture is provided in Figure 1.

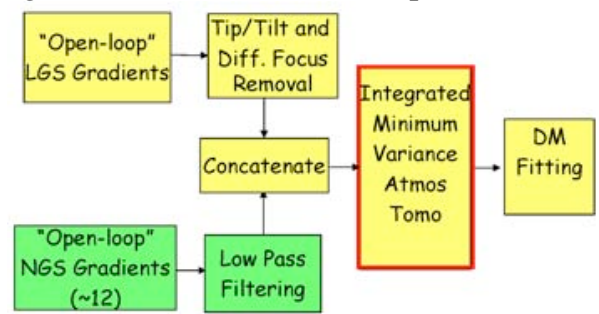

Figure 1. Schematic block diagram of integrated minimum variance atmospheric tomography with joint minimum variance LGS and NGS mode estimation, applicable to all laser tomography adaptive optics systems.

While this formulation is applicable to all laser tomography systems, it requires (i) pre-filtering of the noisy low frame rate NGS measurements in order to optimize servo compensation, (ii) efficient and accurate joint estimation of both high- and low-order atmospheric modes, and (iii) efficient joint 
finite and infinite range ray-tracing operations (contained in the matrices $G_{x, \lg s}$ and $G_{x, n g s}$ ). These practical considerations indicate that a split architecture with separate LGS and NGS control loops is preferred. A step toward the development of such an architecture was recently made by the authors in the context of MCAO, and is briefly reviewed in the next Section.

\section{3 "Ad hoc" Split Tomography Architecture for MCAO}

This architecture implements two separate control loops with distinct wavefront estimation algorithms and temporal filters. The LGS loop is driven by a minimum variance estimator applied to the POL LGS WFS measurements, whereas the NGS loop uses an "ad hoc" least-squares modal estimator applied to the CL NGS WFS measurements and controlling TT and 3 quadratic field distortion modes applied on $2 \mathrm{DMs}$ at different conjugate ranges and scaled so that the wavefront propagated from each LGS to the aperture-plane consists only of pure piston and TT, which are invisible to the TT-removed LGS WFSs [3]. In order to reduce the coupling of the LGS loop into the NGS loop, a rank-5 NGS mode removal projector is applied at the output of the LGS DM fitting step. Denoting by $U$ the rank-5 NGS modal matrix in actuator space, and by $U_{G}=G_{a, n g s} U$ its image onto NGS gradient space, the architecture can be described by the following model:

$$
\begin{array}{ccc}
\hat{x}_{\lg s}=A_{\lg s}^{-1} b_{\lg s}^{P O L}, & y_{l g s}=H_{x} \hat{x}_{l g s}, & q_{l g s}=F y_{l g s}, \\
a_{o, \lg s}=q_{l g s}-U\left(U^{\dagger} q_{l g s}\right), & e_{\lg s}=a_{o, \lg s}-a_{\lg s}, & a_{l g s}(z)=g_{l g s}(z) e_{l g s}(z), \\
c_{n g s}=U_{G}^{\dagger} s_{n g s}^{C L}, & c_{n g s}^{f}(z)=g_{n g s}(z) c_{n g s}(z), & a_{n g s}(z)=U c_{n g s}^{f}(z),
\end{array}
$$

where $U^{\dagger}=\left(U^{T} U\right)^{-1} U^{T}$ and $U_{G}^{\dagger}=\left(U_{G}^{T} C_{n g s}^{-1} U_{G}\right)^{-1} U_{G}^{T} C_{n g s}^{-1}$. The final DM commands are obtained by summing the LGS and NGS commands, i.e. $a=a_{l g s}+a_{n g s}$.

A block diagram illustrating this concept is shown in Figure 2.
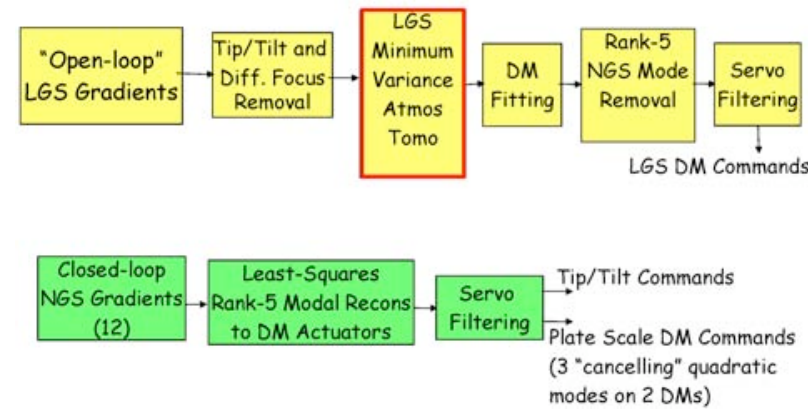

Figure 2 Schematic block diagram of the "ad hoc" split tomography architecture for MCAO.

The main benefits of this approach are: (i) tomographic minimum variance LGS mode estimation using only LGS operators (which relaxes somewhat computational requirements), and (ii) separate NGS mode estimation and servo compensation easy to update for each new NGS asterism. However, on account of the ad hoc definition and control of the NGS modes, this architecture is inappropriate for LTAO or MOAO, and is susceptible to alias the LGS DM commands into the NGS loop, which can result in degraded NGS mode compensation (observed in Monte Carlo simulations of NFIRAOS with the NGS loop running at high frame rate for certain bright NGS asterisms containing stars well outside the DM fitting field of view). To overcome these limitations, a novel split architecture has been designed, which will be presented in the next Section. 


\section{Split Minimum Variance Wavefront Estimation}

A split formulation of the minimum variance estimator (3) can be derived by invoking the ShermanMorrison matrix inversion formula [8], widely applied in real-time Kalman filtering and recursive least-squares methods. Recognizing that $A_{n g s}$ in (4) is a low-rank matrix of rank equal to the total number of NGS WFS measurements (typically 12 for a system comprising 2 TT and 1 tip/tilt/focus/astigmatism (TTFA) WFSs), application of the inversion formula to $A_{\lg s}+A_{n g s}$ allows us to rewrite (3) as follows:

$$
\begin{aligned}
& \hat{x}=\hat{x}_{\lg s}+\hat{x}_{n g s}, \hat{x}_{\lg s}=A_{\lg s}^{-1} b_{\lg s}^{(P) O L}, \hat{x}_{n g s}=E_{n g s}\left(s_{n g s}^{(P) O L}-G_{x, n g s} \hat{x}_{\lg s}\right), \\
& E_{n g s}=U V^{-1}, U=A_{\lg s}^{-1} G_{x, n g s}^{T} C_{n g s}^{-1 / 2}, V=U_{G}+C_{n g s}^{1 / 2}, U_{G}=G_{x, n g s} U .
\end{aligned}
$$

The split formulation (7) is thus analytically equivalent to the standard integrated formulation (3) in the limit of an accurate LGS tomographic solution $\hat{x}_{\lg s} . E_{n g s}$ defines the low-rank NGS tomographic minimum variance estimator, the columns of $U$ are the optimal NGS modes, and those of $U_{G}$ their images onto NGS gradient space. $U$ is pre-computed off-line by Cholesky factorization of $A_{\text {lgs }}$ followed by back-substitutions and can be updated on time scales of tens of seconds as atmospheric conditions or NGS asterism change. A block diagram illustrating this concept is provided in Figure 3.

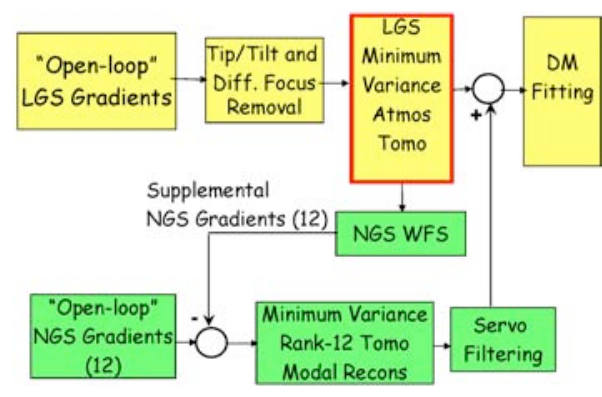

Figure 3 Schematic block diagram of the split atmospheric tomography architecture with separate minimum variance LGS and NGS mode control, applicable to all laser tomography systems.

In practice, servo compensation will be more conveniently performed on a set of orthonormalized modes defined in the aperture-plane as $Q^{\prime}=Q\left(Q^{T} \mathrm{~W} Q\right)^{-1 / 2}$ where $Q=H_{x} U$, so that a more practical scheme may take the following form:

$$
\begin{array}{cl}
c_{n g s}=V^{1-1} s_{n g s}^{(P) O L}, & c_{n g s}^{f}(z)=g_{n g s}^{(1)}(z) c_{n g s}(z), \\
d_{n g s}=V^{\prime-1} G_{x, l g s} \hat{x}_{l g s}, & d_{n g s}^{f}(z)=g_{n g s}^{(2)}(z) d_{n g s}(z), \\
a_{n g s}(z)=Q^{\prime \prime}\left(c_{n g s}^{f}(z)+d_{n g s}^{f}(z)\right), & a(z)=a_{n g s}(z)+a_{l g s}(z) .
\end{array}
$$

where $\quad V^{\prime}=V\left(Q^{T} \mathrm{~W} Q\right)^{-1 / 2}$ and $Q^{\prime \prime}=F Q^{\prime}$. Alternatively, vectors $y_{l g s}=H_{x} \hat{x}_{l g s}$ and $y_{n g s}(z)=Q^{\prime} c_{n g s}^{f}(z)$ can be summed prior to the fitting operation $F$, which avoids computing $Q^{\prime \prime}$.

\section{Sample Monte Carlo Simulation Results for NFIRAOS}

We have conducted side-by-side time domain Monte Carlo simulations for NFIRAOS (order 60 x 60 MCAO system for TMT) to assess the total piston-removed root-mean-square (RMS) wavefront 
error (WFE) for the 3 architectures discussed in this paper. 30 iterations of the CG algorithm were used as tomography solver for all 3 architectures. The 6 LGS WFSs of NFIRAOS were simulated as physical optics WFSs with pre-calculated short-exposure constrained matched filters [9], whereas the 3 NGS WFSs were simulated as geometric Zernike-tilt WFSs. 6 different randomly chosen NGS asterisms within the NFIRAOS 2 arcmin diameter patrol field were selected to run these simulations. Both LGS and NGS loops were synchronized at a common $800 \mathrm{~Hz}$ given the low noise-equivalent angle (NEA) on the NGS WFSs, namely 0.7 mas on the TTFA WFS and 0.35 mas on the two TT WFSs. In order to optimize NGS sharpening over the 2 arcmin patrol field, the DM fitting field was selected to be circular with a 30 arcsec diameter, and performance has been quantified over the NFIRAOS-fed IRIS imager field of view (15 arcsec wide, centered on-axis). All temporal filters were standard type-1 integrators with 2 frames latency of the form $g(z)=\delta /\left(z^{2}-\alpha z-\beta\right)$ with gain $\delta$ chosen equal to 0.5 and actuator weights $\alpha$ and $\beta$ equal to 0.5 each (lossless integrators). Wavefront variances were averaged starting at simulation time step 200 up to time step 4000 (4.75 seconds of real-time). The median piston-removed WFE over the 6 NGS asterisms for the "ad hoc" split MCAO architecture was $193 \mathrm{~nm}$ (including $116 \mathrm{~nm}$ of budgeted implementation errors). The new and integrated architectures reduced this value by $\underline{54 \mathrm{~nm}}$ and $\underline{48 \mathrm{~nm}}$ respectively (most of this improvement being concentraded in the NGS modes).

\section{Conclusions}

We have introduced a novel, computationally efficient, and practical atmospheric tomography wavefront control architecture with separate minimum variance laser and natural guide star mode estimation. The architecture is applicable to all laser tomography systems. Monte Carlo simulations for the TMT MCAO system (NFIRAOS) demonstrate its benefit over a previously introduced "ad hoc" split MCAO architecture, calling for further in-depth analysis and simulations over a representative ensemble of NGS asterisms with optimized NGS loop frame rates and modal gains.

\section{References}

1. “Adaptive Optics Systems," N. N. Hubin, C. E. Max and P. L. Wizinowich, eds, Proc. Soc. Photo-Opt. Instrum. Eng. 7015, entire volume (2008).

2. C. Petit, J.-M. Conan, C. Kulcsar and H. F. Raynaud, "Linear quadratic Gaussian control for adaptive optics and multiconjugate adaptive optics: experimental and numerical analysis," J. Opt. Soc. Am. A 26, 1307-1325 (2009).

3. L. Gilles and B. L. Ellerbroek, "Split atmospheric tomography using laser and natural guide stars," J. Opt. Soc. Am. A 25, 2427-2435 (2008).

4. B. L. Ellerbroek and F. Rigaut, "Methods for correcting tilt anisoplanatism in laser-guide-starbased multiconjugate adaptive optics," J. Opt. Soc. Am. A 18, 2539-2547 (2001).

5. L. Wang, B. L. Ellerbroek and J.-P. Veran, "High fidelity sky coverage analysis via time domain adaptive optics simulations," Appl. Opt. 48, 5076-5087 (2009).

6. T. Pfrommer, P. Hickson and C.-Y. She, "A large-aperture sodium fluorescence lidar with very high resolution for mesopause dynamics and adaptive optics studies," Geophys. Res. Lett. 36, L15831 (2009).

7. B. L. Ellerbroek, "Efficient computation of minimum variance wavefront reconstructors using sparse matrix techniques," J. Opt. Soc. Am. A 19, 1803-1816 (2002).

8. G. H. Golub and C. F. Van Loan, "Matrix Computations," $3^{\text {rd }}$ ed., (The Johns Hopkins University Press, 1996).

9. L. Gilles and B. L. Ellerbroek, "Constrained matched filtering for extended dynamic range and improved noise rejection Shack-Hartmann wavefront sensing," Opt. Lett. 33, 1159-1161 (2008). 